\title{
Gaussian copula of stable random vectors and application
}

\author{
Phuc Ho Dang*1 (D), Truc Giang Vo Thi ${ }^{2}$ (D) \\ ${ }^{1}$ Institute of Mathematics - VAST 18 Hoang Quoc Viet, Ha Noi, Vietnam \\ ${ }^{2}$ Tien Giang University 119 Ap Bac, My Tho City, Vietnam
}

\begin{abstract}
In this paper, we present a new method to investigate data of multivariate heavy-tailed distributions. We show that for any given number $\alpha \in(0 ; 2]$, each Gaussian copula is also the copula of an $\alpha$-stable random vector. Simultaneously, every random vector is $\alpha$-stable if its marginals are $\alpha$-stable and its copula is a Gaussian copula. The result is used to build up a formula representing density functions of $\alpha$-stable random vectors with Gaussian copula. Adopting a new tool, the paper points out that pairs of GPS signals recording latitude and longitude of a fixed point have two-dimensional stable distribution, and in the most of cases, vectors of daily returns in stock market data have multivariate stable distributions with Gaussian copulas.
\end{abstract}

Mathematics Subject Classification (2010). 60E07, 60F05, 60F10, 62E10, 62H05

Keywords. stable distributions, multivariate density function, GPS data, stock market, portfolio selection

\section{Introduction}

Until the 1970's, most of the statistical analysis methods were developed under normality assumptions, mainly for mathematical convenience. In applications, however, normality is only a poor approximation of reality. In particular, normal distributions do not allow heavy tails, which are so common, especially in finance and risk management studies $[4,8,11,14,16,18]$. Arising as solutions to central limit problems, stable distributions are natural heavy tailed extensions of normal distributions and have attracted a lot of attention $[1,2,11,12]$.

While the univariate stable distributions are now mostly accessible by several methods to estimate stable parameters and reliable programs to compute stable densities, cumulative distribution functions, and quantiles for stable random variables $[1,6,10,13]$, the use of the heavy tailed models in practice has been restricted by the lack of the tools for multivariate stable distributions.

The main challenge of dealing with multivariate data with heavy tailed distributions is that of ambiguous dependence between coordinates of a random vector. Whilst the

\footnotetext{
*Corresponding Author.

Email addresses: hdphuc@math.ac.vn (P. Ho Dang), vothitrucgiang@tgu.edu.vn (T. G. Vo Thi)

Received: 19.09.2019; Accepted: 02.03.2020
} 
dependence can be completely determined by covariance matrix for the case of multinormal data, the covariance matrix does not exist for heavy tailed data.

Fortunately, the problem can be solved by the tool of copula. The term copula was first introduced by Sklar [17], but was not of great interest until recent years. Copula functions describe the dependence structure connecting random variables, giving an opportunity to separate the dependence structure and marginal distributions.

Another way of parameterizing multivariate stable distributions is to use the well known univariate stable distribution results about one-dimensional projections of random vectors. However, in practice, this approach faces with challenging computational problems which have not been generally solved for multivariate stable distributions. The problems are caused by the complexity of the possible distributions with an uncountable set of parameters.

In recent years, computations are more accessible for elliptically contoured stable distributions [15] which are scale mixtures of multivariate normal distributions. The tools for the very special class of stable distributions were applied in several empirical studies $[9,12]$. Although the method is available only for a narrow subclass of symmetric multivariate stable distributions, that approach stimulates researchers to create similar tools for other subclasses of general stable multidimensional distributions.

The current paper attempts to develop a new method for investigating the data of multivariate distributions with heavy tails, trying to decrease the complexity of stable copulas downwards to a more practicable case of Gaussian copulas.

The paper is organized as follows. Section 1 presents some auxiliary results on onedimensional stable distributions and copulas. In Section 2 we give the main results of multidimensional stable distributions with Gaussian copulas, demonstrating that Gaussian copulas are also those of some multivariate stable distributions and a random vector is stable if it has Gaussian copula and all its marginals are stable. In the last section we formulate the density function of a stable random vector with Gaussian copula, which can be practically computed. Then the results are applied for studies of GPS data and stock market data.

\section{Preliminaries and notation}

Given a random vector $\mathbf{X}=\left(X_{1}, \ldots, X_{n}\right)^{T}$ taking values in Euclidean space $\mathbb{R}^{n}$, its cumulative distribution function (cdf hereafter) and probability density function (pdf hereafter) are denote by $F_{\mathbf{X}}$ and $f_{\mathbf{X}}$, respectively. The coordinates $X_{1}, \ldots, X_{n}$ are called marginals, simultaneously $F_{X_{1}}, \ldots, F_{X_{n}}$ and $f_{X_{1}}, \ldots, f_{X_{n}}$ are called marginal $c d f$ 's and marginal $p d f$ 's of $\mathbf{X}$, respectively.

A continuous random vector $\mathbf{X}$ is said to have normal distribution ( $\mathbf{X}$ is called a Gaussian or normal random vector) and denoted by $\mathbf{X} \sim N(\boldsymbol{\mu} ; \Sigma)$ if its pdf is given by the formula

$$
f_{\mathbf{X}}(\boldsymbol{x})=\frac{1}{(2 \pi)^{n / 2} \sqrt{|\Sigma|}} \exp \left[-\frac{1}{2}(\boldsymbol{x}-\boldsymbol{\mu})^{T} \Sigma^{-1}(\boldsymbol{x}-\boldsymbol{\mu})\right]
$$

with parameter $\boldsymbol{\mu} \in \mathbb{R}^{n}$ and positive definite matrix $\Sigma \in \mathbb{R}^{n \times n}$. For the special case of standard normal random variable $X \sim N(0 ; 1)$, which plays an important role in statistics, symbols $\varphi$ and $\Phi$ refer to its pdf and cdf, respectively. Then

$$
\varphi(x)=\frac{1}{\sqrt{2 \pi}} \exp \left(-\frac{x^{2}}{2}\right) .
$$

By definition, a random vector $\mathbf{X}$ has stable distribution if for every pair $\left(\mathbf{X}^{\prime}, \mathbf{X}^{\prime \prime}\right)$ of independent random vector's identically distributed as $\mathbf{X}$, for every pair $(a, b)$ of positive numbers, there always exist a positive number $c$ and a vector $\mathbf{d} \in \mathbb{R}^{n}$ such that $a \mathbf{X}^{\prime}+b \mathbf{X}^{\prime \prime}$ 
has the same distribution as $c \mathbf{X}+\mathbf{d}$. It is shown that the constant $c$ is uniquely determined by the pair $(a, b)$. Namely, there is a number $\alpha \in(0 ; 2]$ called stability index satisfied the equation $a^{\alpha}+b^{\alpha}=c^{\alpha}$. Then $\mathbf{X}$ is said to be $\alpha$-stable. Moreover, it is well known that every stable random variable is absolutely continuous with respect to Lebesgue measure on $\mathbb{R}$ and its pdf has a support of the forms $(-\infty ;+\infty),[c ;+\infty)$ or $(-\infty ; c]$ with some $c \in \mathbb{R}$ (see [13], Theorem 1.9 and Lemma 1.10, for instance). Besides, every 2-stable random variable is normally distributed.

From the definition it can be concluded that the stability of a random vector is conserved after any linear transformation. Specifically, if a random vector $\mathbf{X}$ is $\alpha$-stable and $\mathbf{A}$ : $\mathbb{R}^{n} \rightarrow \mathbb{R}^{n}$ is a linear transformation then the random vector $\mathbf{A X}$ is also $\alpha$-stable.

In the one-dimensional case, for all $\alpha \in(0 ; 2]$, every $\alpha$-stable random variable has a characteristic function of the form

$$
\hat{X}(u)=\mathbb{E} \exp (i u X)= \begin{cases}\exp \left(-\gamma^{\alpha}|u|^{\alpha}\left[1-i \beta\left(\tan \frac{\pi \alpha}{2}\right) \operatorname{sign}(u)\right]+i \delta u\right) & \alpha \neq 1 \\ \exp \left(-\gamma|u|\left[1+i \beta \frac{2}{\pi} \operatorname{sign}(u) \ln |u|\right]+i \delta u\right) & \alpha=1,\end{cases}
$$

with fixed $\beta \in[-1 ; 1], \gamma>0$ and $\delta \in \mathbb{R}$. Then the parameters $\alpha, \beta, \gamma$, and $\delta$ uniquely determine the distribution of $X$, the symbol $X \sim \mathbf{S}(\alpha ; \beta ; \gamma ; \delta)$ can be used to refer to that situation. Proposition 1.17 of [13] gives formulas on the parameter's change after a linear transformation. In particular,

Proposition 2.1. (a) If $X \sim \mathbf{S}(\alpha ; \beta ; \gamma ; \delta)$, then for any $a \neq 0$ and $b \in \mathbb{R}$,

$$
a X+b \sim \begin{cases}\mathbf{S}(\alpha ; \operatorname{sign}(a) \beta ;|a| \gamma ; a \delta+b) & \alpha \neq 1 \\ \mathbf{S}\left(1 ; \operatorname{sign}(a) \beta ;|a| \gamma ; a \delta+b-\frac{2}{\pi} \beta a \ln |a|\right) & \alpha=1,\end{cases}
$$

(b) If $X_{1} \sim \mathbf{S}\left(\alpha ; \beta_{1} ; \gamma_{1} ; \delta_{1}\right)$ and $X_{2} \sim \mathbf{S}\left(\alpha ; \beta_{2} ; \gamma_{2} ; \delta_{2}\right)$ are independent, then $X_{1}+X_{2} \sim$ $\mathbf{S}(\alpha ; \beta ; \gamma ; \delta)$, where

$$
\beta=\frac{\beta_{1} \gamma_{1}^{\alpha}+\beta_{2} \gamma_{2}^{\alpha}}{\gamma_{1}^{\alpha}+\gamma_{2}^{\alpha}}, \gamma^{\alpha}=\gamma_{1}^{\alpha}+\gamma_{2}^{\alpha}, \quad \delta=\delta_{1}+\delta_{2} .
$$

For a cdf $G: \mathbb{R} \rightarrow[0 ; 1]$ let $G^{\leftarrow}(y)=\inf \{x: G(x) \geq y\}$ be its generalized inverse. Then copula of a random vector $\mathbf{X}$, denoted by $C_{\mathbf{X}}$, can be defined by

$$
C_{\mathbf{X}}\left(t_{1}, \ldots, t_{n}\right)=F_{\mathbf{X}}\left(F_{X_{1}}^{\overleftarrow{ }}\left(t_{1}\right), \ldots, F_{X_{n}}^{\overleftarrow{ }}\left(t_{n}\right)\right)
$$

for $0 \leq t_{1}, \ldots, t_{n} \leq 1$. The famous Sklar's Theorem (see [17]) confirms the relationship

$$
F_{\mathbf{X}}\left(x_{1}, \ldots, x_{n}\right)=C_{\mathbf{X}}\left(F_{X_{1}}\left(x_{1}\right), \ldots, F_{X_{n}}\left(x_{n}\right)\right),
$$

for $x_{1}, \ldots, x_{n} \in \overline{\mathbb{R}}=[-\infty ;+\infty]$.

When the random vector $\mathbf{X}$ is continuous, its pdf $f_{\mathbf{X}}$ and marginal pdf's $f_{X_{1}}, \ldots, f_{X_{n}}$ exist, simultaneously $F_{X_{k}}^{\overleftarrow{ }}=F_{X_{k}}^{-1}$ for $k=1, \ldots, n$. Then the copula density $c_{\mathbf{X}}$ can be defined and is given by the formula

$$
c_{\mathbf{X}}\left(t_{1}, \ldots, t_{n}\right)=\frac{f_{\mathbf{X}}\left(F_{X_{1}}^{-1}\left(t_{1}\right), \ldots, F_{X_{n}}^{-1}\left(t_{n}\right)\right)}{f_{X_{1}}\left(F_{X_{1}}^{-1}\left(t_{1}\right)\right) \ldots f_{X_{n}}\left(F_{X_{n}}^{-1}\left(t_{n}\right)\right)} .
$$

Besides, the pdf $f_{\mathbf{X}}$ can be calculated from $c_{\mathbf{X}}$ by the identity

$$
f_{\mathbf{X}}\left(x_{1}, \ldots, x_{n}\right)=c_{\mathbf{X}}\left(F_{X_{1}}\left(x_{1}\right), \ldots, F_{X_{n}}\left(x_{n}\right)\right) \times f_{X_{1}}\left(x_{1}\right) \ldots f_{X_{n}}\left(x_{n}\right) .
$$

In general, the copula functions are invariant under strictly increasing transformations. Especially, the following proposition given by Embrechts et al. (see Proposition 5.6[3]) provides an useful tool for getting the main results of this study.

Proposition 2.2. Let $C$ be the copula of a random vector $\mathbf{X}=\left(X_{1}, \ldots, X_{n}\right)^{T}$ and suppose that all marginals $X_{1}, \ldots, X_{n}$ are continuous random variables. If $T_{1}, \ldots, T_{n}$ are strictly increasing functions, then $C$ is also the copula of random vector $\left(T_{1}\left(X_{1}\right), \ldots, T_{n}\left(X_{n}\right)\right)^{T}$. 
The Gaussian copula is the most popular one in applications. It is simply derived from the correlation matrix of a multivariate Gaussian distribution function. For instance, the copula of any two-dimensional Gaussian random vector is completely determined by the correlation coefficient $\rho$ between its marginals through the formula

$$
C(u, v ; \rho)=\frac{1}{2 \pi \sqrt{1-\rho^{2}}} \int_{-\infty}^{\Phi^{-1}(u)} \int_{-\infty}^{\Phi^{-1}(v)} \exp \left(\frac{-\left(s^{2}-2 \rho s t+t^{2}\right)}{2\left(1-\rho^{2}\right)}\right) d s d t
$$

for $(u, v) \in[0 ; 1]^{2}$.

While Gaussian copulas have evidently analytical forms as the above, it raises a question: whether Gaussian copulas can be the copulas of some non - Gaussian stable random vectors or not? Then, the formulas (1.2), (1.3) and (1.4) can be combined together with correlation matrices of Gaussian random vectors to compute pdf's and cdf's of those stable random vectors.

To answer that question, Theorem 2.5 in the next section points out that indeed some stable random vectors have Gaussian copulas. On the other hand, Theorem 2.6 confirms that a random vector $\mathbf{X}=\left(X_{1}, \ldots, X_{n}\right)^{T}$ is $\alpha$-stable with some $\alpha \in(0 ; 2)$ if all marginals $X_{1}, \ldots, X_{n}$ are $\alpha$-stable random variables and the copula $C_{\mathbf{X}}$ is a Gaussian copula. That means possessing Gaussian copula is a sufficient condition for a random vector with stable marginals to be stable itself.

\section{Stable random vectors with Gaussian copula}

Before main results are stated, we formulate some auxiliary lemmas.

Lemma 3.1. Let $Y$ and $Z$ be continuous random variables with pdf's $f_{Y}$ and $f_{Z}$ which are positive on the images $\operatorname{ran}(Y)$ and $\operatorname{ran}(Z)$ of $Y$ and $Z$. Then there exists a strictly increasing function $g: \operatorname{ran}(Y) \rightarrow \operatorname{ran}(Z)$ such that the random variable $g \circ Y: \Omega \rightarrow \operatorname{ran}(Z)$ has the same distribution as $Z$. Moreover, the function $g$ has positive derivative $g^{\prime}$.

Proof. By assumption, the pdf's $f_{Y}$ and $f_{Z}$ are positive, therefore the cdf's $F_{Y}$ and $F_{Z}$ are strictly increasing on $\operatorname{ran}(Y)$ and $\operatorname{ran}(Z)$, respectively. Then the function $g: \operatorname{ran}(Y) \rightarrow$ $\operatorname{ran}(Z)$ defined by $g(u)=F_{Z}^{-1}\left(F_{Y}(u)\right)$ is well-determined as a strictly increasing function. Besides, for each $u \in \operatorname{ran}(Z)$,

$$
g^{\prime}(u)=\frac{f_{Y}(u)}{f_{Z}(g(u))},
$$

which is a positive function. The identity implies

$$
f_{Z}(g(u)) g^{\prime}(u) d u=f_{Y}(u) d u
$$

that yields

$$
F_{Z}(g(u))=\int_{-\infty}^{g(u)} f_{Z}(g(u)) g^{\prime}(u) d u=\int_{-\infty}^{u} f_{Y}(u) d u=F_{Y}(u) .
$$

On the other hand, for every $t \in \mathbb{R}$,

$$
F_{g \circ Y}(t)=\mathbb{P}\{\omega: g(Y(\omega)) \leq t\}=\mathbb{P}\left\{\omega: Y(\omega) \leq g^{-1}(t)\right\}=F_{Y}\left(g^{-1}(t)\right) .
$$

Compared the above with $(2.1)$, putting $t=g(u)$ implies

$$
F_{Z}(t)=F_{g \circ Y}(t) .
$$

This confirm the two random variables $Z$ and $g \circ Y$ have the same distribution. The lemma is proved. 
Although the lemma seems to be quite trivial and simple, it can be useful in practical application. Namely in applied statistics, sometimes normalizing transformations that turn a given data set to a new form with normal distribution need to be used. One of those normalizing transformation for continuously distributed data is proposed in the following immediate consequence of the lemma.

Corollary 3.2. Let $X$ be continuous random variable with $p d f f_{X}$ which is positive on $\operatorname{ran}(X)$. Then there exists a strictly increasing function $g: \operatorname{ran}(X) \rightarrow \mathbb{R}$ such that the random variable $g \circ X$ has normal distribution.

It is evident that all marginals of a stable random vector are stable random variables. The inverse statement is not true, a random vector with all stable marginals is not always stable. However, as it is confirmed in the next lemma, the inverse statement is valid if those marginals are independent. Proof of that is quite simple and need not be presented.

Lemma 3.3. Let $\alpha \in(0 ; 2]$ and a random vector $\mathbf{U}=\left(U_{1}, \ldots, U_{n}\right)^{T}$ be given. Supposed that the marginals $U_{1}, \ldots, U_{n}$ are independent $\alpha$-stable random variables, then $\mathbf{U}$ is an $\alpha$-stable random vector.

The above lemma shows that the independence is a strong condition that guarantees the stability of a random vector with stable marginals. However, for every 2-stable random vector, it is always possible to rotate the space axes to get a new basis, in which the random vector has independent marginals. Namely, the standard Cholesky decomposition theorem immediately implies the following

Lemma 3.4. Let $\mathbf{X}$ be an $n$-dimensional normally distributed random vector with positive defined covariance matrix $\Sigma, \mathbf{X} \sim N_{n}(\boldsymbol{\mu}, \Sigma)$. Then there exists an orthogonal $n \times n$ matrix $A=\left(a_{i j}\right)$ such that the r.v. $\mathbf{Y}=\mathbf{A X}$ has independent normally distributed marginals, where $\mathbf{A}: \mathbb{R}^{n} \rightarrow \mathbb{R}^{n}$ is the linear transformation defined by $\mathbf{A x}=A \mathbf{x}^{T}$.

We are now ready to state the main result.

Theorem 3.5. Let $C$ be a Gaussian copula of a normally distributed random vector $\mathbf{X}$ with positive defined covariance matrix. Then for every number $\alpha \in(0 ; 2]$ there exists an $\alpha$-stable random vector $\mathbf{W}$ such that $C$ is also the copula of $\mathbf{W}$.

Proof. Because both addition and multiplication by positive numbers are strictly increasing transformations in $\mathbb{R}$, by virtue of Proposition 1.2 it can be supposed that all marginals $X_{1}, \ldots, X_{n}$ of the random vector $\mathbf{X}$ are standard normal random variables, $X_{k} \sim N(0 ; 1)$ for $k=1, \ldots, n$.

Based on the assumption, the covariance matrix $\Sigma$ of $\mathbf{X}$ is positive defined, Lemma 2.4 implies the existence of an orthogonal $n \times n$ matrix $A=\left(a_{i j}\right), A^{-1}=A^{T}$, such that the normal random vector $\mathbf{Y}=A \mathbf{X}$ has independent marginals $Y_{1}, \ldots, Y_{n}$ and diagonal covariance matrix $A \Sigma A^{T}$ with diagonal elements consist of all eigenvalues of $\Sigma$.

Now $\alpha$-stable random variables $S_{1}, \ldots, S_{n}$ are concerned. Lemma 2.1 ensures that, for each $k=1, \ldots, n$, there exists a strictly increasing function $g_{k}: \mathbb{R} \rightarrow \operatorname{ran}\left(S_{k}\right)$ such that the random variable $U_{k}=g_{k} \circ Y_{k}$ has the same $\alpha$-stable distribution as $S_{k}$. Simultaneously, the independence of marginals $Y_{1}, \ldots, Y_{n}$ implies the independence of random variables $U_{1}, \ldots, U_{n}$. Thus, the random vector $\mathbf{U}=\left(U_{1}, \ldots, U_{n}\right)^{T}$ has independent $\alpha$-stable marginals, it must be an $\alpha$-stable random vector as the conclusion of Lemma 2.3.

Let define a new random vector $\mathbf{W}=\left(W_{1}, \ldots, W_{n}\right)^{T}=A^{-1} \mathbf{U}$. Then it is clear that $\mathbf{W}$ is also an $\alpha$-stable random vector as $\mathbf{U}$. We attempt to point out that $\mathbf{W}$ has the same copula $C$ as $\mathbf{X}$. Firstly, the $\alpha$-stability of marginals $W_{1}, \ldots, W_{n}$ of the random vector $\mathbf{W}$ together with Lemma 2.1 ensures that for each $k=1, \ldots, n$, there exist strictly increasing function $h_{k}$ such that the random variable $Z_{k}=h_{k} \circ W_{k}$ has standard normal distribution 
as $X_{k}$. Consequently, due to Proposition 1.2 , the random vector's $\mathbf{W}$ and $\mathbf{Z}=\left(Z_{1}, \ldots, Z_{n}\right)^{T}$ have the same copula, $C_{\mathbf{W}}=C_{\mathbf{Z}}$. Therefore, to complete the proof, it is sufficient to show that $C_{\mathbf{Z}}=C_{\mathbf{X}}$, which is equivalent to

$$
c_{\mathbf{X}}=c_{\mathbf{Z}}
$$

However, for $\left(u_{1}, \ldots, u_{n}\right) \in[0 ; 1]^{n}$, it implies from (1.3) that

$$
\begin{gathered}
c_{\mathbf{X}}\left(u_{1}, \ldots, u_{n}\right)=\frac{f_{\mathbf{X}}\left(F_{X_{1}}^{-1}\left(u_{1}\right), \ldots, F_{X_{n}}^{-1}\left(u_{n}\right)\right)}{f_{X_{1}}\left(F_{X_{1}}^{-1}\left(u_{1}\right)\right) \ldots f_{X_{n}}\left(F_{X_{n}}^{-1}\left(u_{n}\right)\right)} \\
=\frac{f_{\mathbf{X}}\left(\Phi^{-1}\left(u_{1}\right), \ldots, \Phi^{-1}\left(u_{n}\right)\right)}{\varphi\left(\Phi^{-1}\left(u_{1}\right)\right) \ldots \varphi\left(\Phi^{-1}\left(u_{n}\right)\right)}
\end{gathered}
$$

and

$$
\begin{gathered}
c_{\mathbf{Z}}\left(u_{1}, \ldots, u_{n}\right)=\frac{f_{\mathbf{Z}}\left(F_{Z_{1}}^{-1}\left(u_{1}\right), \ldots, F_{Z_{n}}^{-1}\left(u_{n}\right)\right)}{f_{Z_{1}}\left(F_{Z_{1}}^{-1}\left(u_{1}\right)\right) \ldots f_{Z_{n}}\left(F_{Z_{n}}^{-1}\left(u_{n}\right)\right)} \\
=\frac{f_{\mathbf{Z}}\left(\Phi^{-1}\left(u_{1}\right), \ldots, \Phi^{-1}\left(u_{n}\right)\right)}{\varphi\left(\Phi^{-1}\left(u_{1}\right)\right) \ldots \varphi\left(\Phi^{-1}\left(u_{n}\right)\right)}
\end{gathered}
$$

From (2.3) and (2.4), it is evident that (2.2) is equivalent to

$$
f_{\mathbf{X}}\left(\Phi^{-1}\left(u_{1}\right), \ldots, \Phi^{-1}\left(u_{n}\right)\right)=f_{\mathbf{Z}}\left(\Phi^{-1}\left(u_{1}\right), \ldots, \Phi^{-1}\left(u_{n}\right)\right) .
$$

Denoting $\mathbf{g}:=\left(g_{1}, \ldots, g_{n}\right), \mathbf{h}:=\left(h_{1}, \ldots, h_{n}\right)$, and

$$
Q:=A^{-1} \circ \mathbf{g} \circ A
$$

we see that $\mathbf{Z}=\mathbf{h}(\mathbf{W})$ and $\mathbf{W}=Q(\mathbf{X})$. Then, due to $h_{k}=\Phi^{-1} \circ F_{W_{k}}$ and the independence of $Y_{1}, \ldots, Y_{n}$, setting $x_{1}=F_{W_{1}}^{-1}\left(u_{1}\right), \ldots, x_{n}=F_{W_{n}}^{-1}\left(u_{n}\right)$, the right hand side of (3.5) equals to

$$
\begin{gathered}
f_{\mathbf{Z}}\left(\Phi^{-1}\left(u_{1}\right), \ldots, \Phi^{-1}\left(u_{n}\right)\right)=f_{\mathbf{h} \circ \mathbf{W}}\left(\Phi^{-1}\left(u_{1}\right), \ldots, \Phi^{-1}\left(u_{n}\right)\right) \\
=f_{\mathbf{W}}\left(\mathbf{h}^{-1}\left(\Phi^{-1}\left(u_{1}\right), \ldots, \Phi^{-1}\left(u_{n}\right)\right)=f_{Q \circ \mathbf{X}}\left(h_{1}^{-1}\left(\Phi^{-1}\left(u_{1}\right)\right), \ldots, h_{n}^{-1}\left(\Phi^{-1}\left(u_{n}\right)\right)\right)\right. \\
=f_{\mathbf{X}}\left(Q^{-1}\left(h_{1}^{-1}\left(\Phi^{-1}\left(u_{1}\right)\right), \ldots, h_{n}^{-1}\left(\Phi^{-1}\left(u_{n}\right)\right)\right)\right) \\
=f_{\mathbf{X}}\left(A^{-1} \circ \mathbf{g}^{-1} \circ A\left(h_{1}^{-1}\left(\Phi^{-1}\left(u_{1}\right)\right), \ldots, h_{n}^{-1}\left(\Phi^{-1}\left(u_{n}\right)\right)\right)\right) \\
=f_{\mathbf{X}}\left(A^{-1} \circ \mathbf{g}^{-1} \circ A\left(F_{W_{1}}^{-1}\left(u_{1}\right), \ldots, F_{W_{n}}^{-1}\left(u_{n}\right)\right)\right) \\
=f_{A^{-1} \circ \mathbf{Y}}\left(A^{-1} \circ \mathbf{g}^{-1} \circ A\left(x_{1}, \ldots, x_{n}\right)\right)=f_{\mathbf{Y}}\left(A \circ A^{-1} \circ \mathbf{g}^{-1} \circ A\left(x_{1}, \ldots, x_{n}\right)\right) \\
=f_{Y_{1}}\left(g_{1}^{-1}\left(\sum_{j=1}^{n} a_{1 j} x_{j}\right)\right) \cdot \ldots \cdot f_{Y_{1}}\left(g_{n}^{-1}\left(\sum_{j=1}^{n} a_{n j} x_{j}\right)\right) \\
=f_{U_{1}}\left(\sum_{j=1}^{n} a_{1 j} x_{j}\right) \cdot \ldots \cdot f_{U_{n}}\left(\sum_{j=1}^{n} a_{n j} x_{j}\right) .
\end{gathered}
$$

Simultaneously, setting $y_{1}=F_{U_{1}}^{-1}\left(u_{1}\right), \ldots, y_{n}=F_{U_{n}}^{-1}\left(u_{n}\right)$, the left hand side of (3.5) is equal to

$$
\begin{gathered}
f_{A^{-1} \circ \mathbf{Y}}\left(\Phi^{-1}\left(u_{1}\right), \ldots, \Phi^{-1}\left(u_{n}\right)\right)=f_{\mathbf{Y}}\left(A\left(\Phi^{-1}\left(F_{U_{1}}\left(y_{1}\right)\right), \ldots, \Phi^{-1}\left(F_{U_{n}}\left(y_{n}\right)\right)\right)\right. \\
=f_{\mathbf{Y}}\left(A\left(g_{1}\left(y_{1}^{-1}\right), \ldots, f_{Y_{n}}\left(g_{n}^{-1}\left(y_{n}\right)\right)\right)\right) \\
=f_{Y_{1}}\left(\sum_{j=1}^{n} a_{1 j} g_{j}^{-1}\left(y_{j}\right)\right) \cdot \ldots \cdot f_{Y_{n}}\left(\sum_{j=1}^{n} a_{n j} g_{j}^{-1}\left(y_{j}\right)\right) \\
=\frac{1}{\sqrt{2 \pi}} \exp \left(-\frac{1}{2}\left[\sum_{j=1}^{n} a_{1 j} g_{j}^{-1}\left(y_{j}\right)\right]^{2}\right) \cdot \ldots \cdot \frac{1}{\sqrt{2 \pi}} \exp \left(-\frac{1}{2}\left[\sum_{j=1}^{n} a_{n j} g_{j}^{-1}\left(y_{j}\right)\right]^{2}\right) \\
=\left(\frac{1}{\sqrt{2 \pi}}\right)^{n} \exp \left(-\frac{1}{2} \sum_{k=1}^{n}\left(\left[\sum_{j=1}^{n} a_{k j} g_{j}^{-1}\left(y_{j}\right)\right]^{2}\right)\right.
\end{gathered}
$$




$$
\begin{gathered}
=\left(\frac{1}{\sqrt{2 \pi}}\right)^{n} \exp \left(-\frac{1}{2} \sum_{k=1}^{n} \sum_{j=1}^{n} a_{k j} g_{j}^{-1}\left(y_{j}\right) \sum_{i=1}^{n} a_{k i} g_{i}^{-1}\left(y_{i}\right)\right) \\
=\left(\frac{1}{\sqrt{2 \pi}}\right)^{n} \exp \left(-\frac{1}{2} \sum_{j=1}^{n} \sum_{i=1}^{n} \sum_{k=1}^{n} a_{k j} a_{k i} g_{j}^{-1}\left(y_{j}\right) g_{i}^{-1}\left(y_{i}\right)\right) \\
=\left(\frac{1}{\sqrt{2 \pi}}\right)^{n} \exp \left(-\frac{1}{2} \sum_{j=1}^{n} \sum_{i=1}^{n} \delta_{j i} g_{j}^{-1}\left(y_{j}\right) g_{i}^{-1}\left(x_{i}\right)\right) \\
=\left(\frac{1}{\sqrt{2 \pi}}\right)^{n} \exp \left(-\frac{1}{2} \sum_{j=1}^{n}\left(g_{j}^{-1}\left(x_{j}\right)\right)^{2}\right)=\varphi\left(g_{1}^{-1}\left(x_{1}\right)\right) \ldots \varphi\left(g_{n}^{-1}\left(y_{n}\right)\right) \\
=f_{Y_{1}}\left(g_{1}^{-1}\left(y_{1}\right)\right) \ldots f_{Y_{n}}\left(g_{n}^{-1}\left(y_{n}\right)\right)=f_{U_{1}}\left(y_{1}\right) \ldots f_{U_{n}}\left(y_{n}\right),
\end{gathered}
$$

where $\delta_{k k}=1$ for $k=1, \ldots, n ; \delta_{k i}=0$ for $k \neq i=1, \ldots, n$. Comparing (3.6) to (3.7), with $y_{1}=\sum_{j=1}^{n} a_{1 j} x_{j}, \ldots, y_{n}=\sum_{j=1}^{n} a_{n j} x_{j}$, we can conclude (3.5) is true, that means (3.2) holds, the proof completes.

The next theorem presents Gaussian copula as a sufficient condition for a random vector with stable marginals to have stable distribution.

Theorem 3.6. For given $\alpha \in(0 ; 2]$ let $\mathbf{X}$ be a Gaussian random vector with positive defined covariance matrix such that the matrix $A=\left(a_{i j}\right)$ defined in the Lemma 2.4 satisfies $\operatorname{det}\left(\left|a_{i j}\right|^{\alpha}\right) \neq 0$. Suppose that $\mathbf{W}^{*}=\left(W_{1}^{*}, \ldots, W_{n}^{*}\right)^{T}$ is a random vector with $\alpha$-stable marginals $W_{1}^{*}, \ldots, W_{n}^{*}$ such that the copulas of $\mathbf{W}^{*}$ and of $\mathbf{X}$ are equal, $C_{\mathbf{W}^{*}}=C_{\mathbf{X}}$. Then $\mathbf{W}^{*}$ is an $\alpha$-stable random vector.

Proof. Let $\beta_{1}^{*}, \ldots, \beta_{n}^{*} \in[-1 ; 1] ; \gamma_{1}^{*}, \ldots, \gamma_{n}^{*}>0$; and $\delta_{1}^{*}, \ldots, \delta_{n}^{*} \in \mathbb{R}$ be the stable parameters and $W_{1}^{*} \sim S\left(\alpha ; \beta_{1}^{*} ; \gamma_{1}^{*} ; \delta_{1}^{*}\right), \ldots, W_{n}^{*} \sim S\left(\alpha ; \beta_{n}^{*} ; \gamma_{n}^{*} ; \delta_{n}^{*}\right)$. Then, since $\alpha$-stability of a random vector is unchanged after invertible linear transformation

$$
\left(x_{1}, \ldots, x_{n}\right) \mapsto\left(a_{1} x_{1}+b_{1}, \ldots, a_{n} x_{n}+b_{n}\right)
$$

for any $a_{1}>0, \ldots, a_{n}>0$ and $\left(b_{1}, \ldots, b_{n}\right) \in \mathbb{R}^{n}$, without loss of generality, it can be supposed that $\gamma_{1}^{*}=\ldots=\gamma_{n}^{*}=1$ and $\delta_{1}^{*}=\ldots=\delta_{n}^{*}=0$.

Let matrix $A=\left(a_{i j}\right)$ and the random vector $\mathbf{Y}=\left(Y_{1}, \ldots, Y_{n}\right)^{T}=A \mathbf{X}$ be determined as in Lemma 2.4. We attempt to determine $\alpha$-stable random variables $S_{1} \sim S\left(\alpha ; \beta_{1} ; \gamma_{1} ; 0\right)$, $\ldots, S_{n} \sim S\left(\alpha ; \beta_{n} ; \gamma_{n} ; 0\right)$ satisfying the equations

$$
\left\{\begin{array}{c}
1=\left(\gamma_{1}^{*}\right)^{\alpha}=\left|a_{11}\right|^{\alpha} \gamma_{1}^{\alpha}+\ldots+\left|a_{n 1}\right|^{\alpha} \gamma_{n}^{\alpha} \\
1=\left(\gamma_{n}^{*}\right)^{\alpha}=\left|a_{1 n}\right|^{\alpha} \gamma_{1}^{\alpha}+\ldots+\left|a_{n n}\right|^{\alpha} \gamma_{n}^{\alpha}
\end{array}\right.
$$

and

$$
\left\{\begin{array}{l}
\beta_{1}^{*}=\frac{\beta_{1}\left|a_{11}\right|^{\alpha} \gamma_{1}^{\alpha}+\ldots+\beta_{n}\left|a_{n 1}\right|^{\alpha} \gamma_{n}^{\alpha}}{\left|a_{11}\right|^{\alpha} \gamma_{1}^{\alpha}+\ldots+\left|a_{n 1}\right|^{\alpha} \gamma_{n}^{\alpha}}=\left|a_{11}\right|^{\alpha} \gamma_{1}^{\alpha} \beta_{1}+\ldots+\left|a_{n 1}\right|^{\alpha} \gamma_{n}^{\alpha} \beta_{n} \\
\beta_{n}^{*}=\frac{\beta_{1}\left|a_{1 n}\right|^{\alpha} \gamma_{1}^{\alpha}+\ldots+\beta_{n}\left|a_{n n}\right|^{\alpha} \gamma_{n}^{\alpha}}{\left|a_{1 n}\right|^{\alpha} \gamma_{1}^{\alpha}+\ldots+\left|a_{n n}\right|^{\alpha} \gamma_{n}^{\alpha}}=\left|a_{1 n}\right|^{\alpha} \gamma_{1}^{\alpha} \beta_{1}+\ldots+\left|a_{n n}\right|^{\alpha} \gamma_{n}^{\alpha} \beta_{n}
\end{array}\right.
$$

with unknowns $\gamma_{1}^{\alpha}, \ldots, \gamma_{n}^{\alpha}$ and $\gamma_{1}^{\alpha} \beta_{1}, \ldots, \gamma_{n}^{\alpha} \beta_{n}$.

From the assumption, $\operatorname{det}\left(\left|a_{i j}\right|^{\alpha}\right) \neq 0$, it is clear that the equations (3.9) and (3.10) are solved and the $\alpha$-stable random variables $S_{1} \sim S\left(\alpha ; \beta_{1} ; \gamma_{1} ; 0\right), \ldots, S_{n} \sim S\left(\alpha ; \beta_{n} ; \gamma_{n} ; 0\right)$ are completely defined. By virtue of Lemma 2.1 , for each $k=1, \ldots, n$, there exists a strictly increasing function $g_{k}: \mathbb{R} \rightarrow \operatorname{ran}\left(S_{k}\right)$ such that the random variable $U_{k}=g_{k} \circ Y_{k}$ has the same $\alpha$-stable distribution as $S_{k}$. Simultaneously, the independence of marginals $Y_{1}, \ldots, Y_{n}$ implies the independence of $\alpha$-stable random variables $U_{1}, \ldots, U_{n}$.

With $\mathbf{W}=\left(W_{1}, \ldots, W_{n}\right)^{T}=A^{-1} \mathbf{U}$, by the same argument of Theorem 2.5, it is certain that $\mathbf{W}$ is an $\alpha$-stable random vector that has the same copula as $\mathbf{X}, C_{\mathbf{W}}=C_{\mathbf{X}}=C_{\mathbf{W}^{*}}$. 
On the other hand, the equations (3.9) and (3.10) together with Proposition 1.1 imply the equality in distribution of all the marginals of $\mathbf{W}$ to correspondent marginals of $\mathbf{W}^{*}$. In particular, $W_{1} \sim S\left(\alpha ; \beta_{1}^{*} ; \gamma_{1}^{*} ; \delta_{1}^{*}\right), \ldots, W_{n} \sim S\left(\alpha ; \beta_{n}^{*} ; \gamma_{n}^{*} ; \delta_{n}^{*}\right)$. Consequently, $\mathbf{W}^{*} \stackrel{D}{=} \mathbf{W}$ and $\mathbf{W}^{*}$ is an $\alpha$-stable random vector, the proof is fulfilled.

The above theorem suggests a procedure to check whether a data set can be fitted to any stable random vector or not, with details as follows:

Step 1. To estimate stable parameters of data marginals and to check if the all marginals have $\alpha$-stable distributions with a common suitably chosen $\alpha$.

Step 2. To estimate a Gaussian copula for the transformed data having normal distributed marginals, and to check if the original data are fitted to that Gaussian copula.

If the two steps are satisfied, it can be concluded the data vector has stable distribution.

\section{Application}

This section proposes a new method to analyze data of stable distribution with Gaussian copula by using the results given in the previous section. The special structure of Gaussian copulas allows researchers to combine well - known computational tools for onedimensional stable distributions and Gaussian copulas to compute density functions and cumulative distribution functions of data which follow stable distributions with Gaussian copulas.

\subsection{Computation with multivariate data of stable distribution}

Nolan[12] represented an approach to determine density functions of stable random vectors belonging to a specific class of random vectors with elliptically contoured stable distributions. At first, the researcher dealt with an $\alpha$-stable radially symmetric (around $\mathbf{0})$ random vector $\mathbf{X}$ with density function $f_{\mathbf{X}}(\mathbf{x})$ and amplitude $R=|\mathbf{X}|$. For the case $0<\alpha<2$, the study showed that

$$
\mathbf{X} \stackrel{D}{=} Z^{1 / 2} \mathbf{G}_{1},
$$

where $Z \sim S\left(\alpha / 2 ; 1 ; 2 \gamma_{0}^{2}(\cos \pi \alpha / 4)^{2 / \alpha} ; 0\right)$ is positive stable and $\mathbf{G}_{1} \sim N(\mathbf{0} ; \mathbf{I}), Z$ and $\mathbf{G}_{1}$ are independent. Then $R^{2} \stackrel{D}{=} Z T$, where $T$ is a chi-squared random variable with $n$ degrees of freedom, and independent of $Z$. From this equation, the density functions of $R$ and $\mathbf{X}$ are completely defined by

$$
f_{R}(r)=f_{R}\left(r \mid \alpha ; \gamma_{0} ; n\right)=2 r \int_{0}^{\infty} f_{Z}\left(r^{2} / t\right) \frac{f_{T}(t)}{t} d t
$$

and

$$
f_{\mathbf{X}}(\mathbf{x})= \begin{cases}\left(\Gamma(n / 2) /\left(2 \pi^{n / 2}\right)\right)|\mathbf{x}|^{1-n} f_{R}\left(|\mathbf{x}| \mid \alpha ; \gamma_{0} ; n\right) & \mathbf{x} \neq \mathbf{0} \\ \Gamma(n / \alpha) /\left(\alpha 2^{n-1} \pi^{n / 2} \Gamma(n / 2)^{2} \gamma_{0}^{n}\right) & \mathbf{x}=\mathbf{0} .\end{cases}
$$

A random vector $\mathbf{Y}$ is called elliptically contoured stable if it is an affine transformation of the $\alpha$-stable radially symmetric random vector $\mathbf{X}$ by $\mathbf{Y}=\Sigma^{1 / 2} \mathbf{X}+\delta$, where $\Sigma^{1 / 2}$ is from the Cholesky decomposition of a positive definite matrix $\Sigma$ and $\delta \in \mathbb{R}^{n}$. Then

$$
\mathbf{Y} \stackrel{D}{=} Z^{1 / 2} \Sigma^{1 / 2} \mathbf{G}_{1}+\delta
$$

and

$$
f_{\mathbf{Y}}(\mathbf{y})=|\operatorname{det} \Sigma|^{-1 / 2} f_{\mathbf{X}}\left(\Sigma^{-1 / 2} \mathbf{y}\right) .
$$

Since the tools for computation of univariate stable density functions are quite commonly available at present, the formulas (4.1), (4.2) and (4.3) make the analyses of data with elliptically contoured stable distributions easier. 
However, it is clear that all marginals of an $\alpha$-stable radially symmetric random vector are identically distributed and all marginals of an elliptically contoured stable random vector are symmetric (around respective location parameter). Those properties of data are quite rarely met in practice. In the following part, we propose a method of computing density functions of stable random vectors having Gaussian copulas. Whilst marginals of a stable random vector with Gaussian copula are not necessarily symmetric, this model of multivariate stable distributions may be more acceptable in the practical data analysis.

Let $\mathbf{G} \sim N(\mathbf{0} ; \Sigma)$ be a Gaussian random vector with covariance matrix $\Sigma=\left(\sigma_{i j}\right)$ and $\sigma_{i i}=1$ for all $i=1, \ldots, n$. It is evident that $\Sigma$ also is the correlation matrix of $\mathbf{G}$ and all marginals of this random vector are standard normal random variables. Then $\mathbf{G}$ has the density function

$$
f_{\mathbf{G}}(\mathbf{x})=(2 \pi)^{-n / 2}|\Sigma|^{-1 / 2} \exp \left(-\frac{1}{2} \mathbf{x} \Sigma^{-1} \mathbf{x}^{T}\right)
$$

for $\mathbf{x}=\left(x_{1}, \ldots, x_{n}\right) \in \mathbb{R}^{n}$, and from (1.1), its copula can be computed by

$$
C_{\mathbf{G}}(\mathbf{u})=\frac{1}{(2 \pi)^{n / 2}|\Sigma|^{1 / 2}} \int_{-\infty}^{\Phi^{-1}\left(u_{1}\right)} \ldots \int_{-\infty}^{\Phi^{-1}\left(u_{n}\right)} \exp \left(-\frac{1}{2} \mathbf{x} \Sigma^{-1} \mathbf{x}^{T}\right) d x_{n} \ldots d x_{1}
$$

for $\mathbf{u}=\left(u_{1}, \ldots, u_{n}\right) \in[0 ; 1]^{n}$. Moreover from (1.3), its copula density is determined by

$$
\begin{gathered}
c_{\mathbf{G}}(\mathbf{t})=\frac{f_{\mathbf{G}}\left(\Phi^{-1}\left(t_{1}\right), \ldots, \Phi^{-1}\left(t_{n}\right)\right)}{\varphi\left(\Phi^{-1}\left(t_{1}\right)\right) \ldots \varphi\left(\Phi^{-1}\left(t_{n}\right)\right)} \\
=\frac{\exp \left(-\frac{1}{2}\left(\Phi^{-1}\left(t_{1}\right), \ldots, \Phi^{-1}\left(t_{n}\right)\right) \Sigma^{-1}\left(\Phi^{-1}\left(t_{1}\right), \ldots, \Phi^{-1}\left(t_{n}\right)\right)^{T}\right)}{(2 \pi)^{n / 2}|\Sigma|^{1 / 2} \varphi\left(\Phi^{-1}\left(t_{1}\right)\right) \ldots \varphi\left(\Phi^{-1}\left(t_{n}\right)\right)}
\end{gathered}
$$

for $\mathbf{t}=\left(t_{1}, \ldots, t_{n}\right) \in[0 ; 1]^{n}$.

For fixed $\alpha \in(0 ; 2)$, let $S_{1} \sim S\left(\alpha ; \beta_{1} ; \gamma_{1} ; \delta_{1}\right), \ldots, S_{n} \sim S\left(\alpha ; \beta_{n} ; \gamma_{n} ; \delta_{n}\right)$ be certain $\alpha$-stable random variables with density functions $f_{S_{1}}, \ldots, f_{S_{n}}$ and cumulative distribution functions $F_{S_{1}}, \ldots, F_{S_{n}}$, respectively. Then formula (1.2) from Sklar's Theorem ensures the function

$$
F_{\mathbf{Y}}\left(y_{1}, \ldots, y_{n}\right)=C_{\mathbf{G}}\left(F_{S_{1}}\left(y_{1}\right), \ldots, F_{S_{n}}\left(y_{n}\right)\right)
$$

for $y_{1}, \ldots, y_{n} \in \overline{\mathbb{R}}=[-\infty ;+\infty]$, defines the cumulative distribution function of some random vector $\mathbf{Y}$ with copula $C_{\mathbf{G}}$ and marginals $S_{1}, \ldots, S_{n}$. In the case when the condition of Theorem 2.6 fulfilled for $A=\Sigma^{1 / 2}$, the random vector $\mathbf{Y}$ is truly an $\alpha$-stable random vector, and by virtue of formula (1.4), its density function has the following form:

$$
\begin{gathered}
f_{\mathbf{Y}}\left(y_{1}, \ldots, y_{n}\right)=c_{\mathbf{G}}\left(F_{S_{1}}\left(y_{1}\right), \ldots, F_{S_{n}}\left(y_{n}\right)\right) \times f_{S_{1}}\left(y_{1}\right) \ldots f_{S_{n}}\left(y_{n}\right) \\
=\frac{\exp \left(-\frac{1}{2}\left(\Phi^{-1} F_{S_{1}}\left(y_{1}\right), \ldots, \Phi^{-1} F_{S_{n}}\left(y_{n}\right)\right) \Sigma^{-1}\left(\Phi^{-1} F_{S_{1}}\left(y_{1}\right), \ldots, \Phi^{-1} F_{S_{n}}\left(y_{n}\right)\right)^{T}\right)}{(2 \pi)^{n / 2}|\Sigma|^{1 / 2} \varphi\left(\Phi^{-1} F_{S_{1}}\left(y_{1}\right)\right) \ldots \varphi\left(\Phi^{-1} F_{S_{n}}\left(y_{n}\right)\right)} \\
\times f_{S_{1}}\left(y_{1}\right) \ldots f_{S_{n}}\left(y_{n}\right) .
\end{gathered}
$$

It is evident that (3.5) can be calculate by ordinary computing without symmetry assumption on the $\alpha$-stable random variables $S_{1}, \ldots, S_{n}$. Therefore, the approach represented here is more flexible than the one of (4.3).

\subsection{Multivariate stable distribution of GPS data}

In this subsection we examine the probability distribution of two-dimensional random vectors of GPS data. The data used in this study are given by Bui Quang[2] and were taken from a GPS receiver device (JMC GP-200) fixed at the window of a room on the 3rd floor in a 5 floor's building. The device was connected with a computer to automatically record data by a software (format-NMEA0183). With the equipment, longitude and latitude coordinates of the fixed location were recorded sequentially every one second. The measurement was conducted three times in May 2011, with the duration of 10 - 15 minutes each time, providing three files with 812,752 , and 627 signals, respectively. Totally, 
we got a data set with 2191 signals, each signal include a pair of longitude and latitude coordinates recorded. Due to the changes of satellites positions on orbits, the measures varied moment by moment though the location was fixed. However, we can determine the distribution of the deviations from the "true" coordinates of the location, which will be done as follows.

Traditionally, deviations in any measurement were treated as values of a normally distributed quantity. Therefore, Kolmogorov - Smirnov test (K-S test) was used to check if the GPS data followed the normal distribution. The results are represented in Table 1, with $(X, Y)$ denoted the pairs of longitude and latitude observed. In this table, the two p-values presented less than 0.05 , rejecting the hypothesis of having normal distribution for coordinates. That means the two-dimensional vectors of longitudes and latitudes are not normally distributed.

Guessing the two-dimensional coordinate vectors have stable distribution, we use the functions McCullochParametersEstim and ks.test in the software $\mathrm{R}$ were used to estimate stable parameters for each coordinate and then do K-S test verifying the hypothesis of stable distribution, taking $\bar{\alpha}$ (the average of two values of $\alpha$ ) as the common first stable parameter. The results are presented in Table 2, where the p-values are greater than 0.05 , indicating every coordinate has stable distribution $S(\bar{\alpha} ; \beta ; \gamma ; \delta)$ with the corresponding parameters. That means

$$
\begin{aligned}
& X \sim S\left(1.567 ;-0.1024 ; 3.15 \cdot 10^{-5} ; 105.799922\right), \\
& Y \sim S\left(1.567 ; 0.1592 ; 2.16 \cdot 10^{-5} ; 21.043174\right) .
\end{aligned}
$$

According to Theorem 2.6, the random vector $(X, Y)$ will be stable if it has Gaussian copula. By Proposition 1.1 and Corollary 2.2, the Gaussian copula of $(X, Y)$ is completely determined by the correlation coefficient $\operatorname{corr}\left(X^{\prime}, Y^{\prime}\right)$, where variables $X^{\prime}$ and $Y^{\prime}$ are standard normal variables transformed from $X$ and $Y$ by

$$
\begin{aligned}
x_{j}^{\prime} & =\Phi^{-1}\left(\hat{F}_{X}\left(x_{j}\right)\right), \\
y_{j}^{\prime} & =\Phi^{-1}\left(\hat{F}_{Y}\left(y_{j}\right)\right),
\end{aligned}
$$

with $j=1, \ldots, N, \hat{F}_{X}$ and $\hat{F}_{Y}$ being the empirical cumulative distribution functions of $X$ and $Y$, respectively. Actually,

$$
\rho=\operatorname{corr}\left(X^{\prime}, Y^{\prime}\right)=-0.01462955 .
$$

We attempt now to show that the copulas defined by (2.5) with the correlation coefficients $\rho$ will be truly the copula of GPS coordinates corresponding to the data vectors $(X, Y)$.

Genest and Rémillard[5] established the validity of the parametric bootstrap method when testing the goodness of-fit of families of multivariate distributions and copulas. Based on the reliable theoretical frame and other related ones, Kojadinovic and Yan[7] built up an $\mathrm{R}$ package named copula to implement the goodness-of-fit tests. Used the function named gofCopula in the copula package, we tested the hypothesis

$$
H_{0}: C_{(X, Y)}(u, v)=C(u, v ; \rho),
$$

$(u, v) \in[0 ; 1]^{2}$, where $C(u, v ; \rho)$ is the Gaussian copula defined by (1.5) with the correlation coefficient $\rho$ given in (4.6).

The testing result approximates p-value of 0.6489 , much greater than 0.05 . That means the hypothesis can be accepted. Then we can confirm that the GPS data vector has Gaussian copula, consequently it has two-dimensional stable distribution by virtue of Theorem 2.6. 
Table 1. Kolmogorov-Smirnov test for normal distribution of GPS data

\begin{tabular}{lllll}
\hline Coordinate & N (Size) & Mean & SD & p-value \\
\hline$X$ & 2191 & 105.799922 & 0.000053 & $4.6 \cdot 10^{-5}$ \\
$Y$ & 2191 & 21.043174 & 0.000039 & $2.4 \cdot 10^{-8}$ \\
\hline
\end{tabular}

Table 2. Parameters and Kolmogorov-Smirnov test for stable distribution

\begin{tabular}{lllllll}
\hline Coordinate & $\alpha$ & $\bar{\alpha}$ & $\beta$ & $\gamma$ & $\delta$ & $\mathrm{p}$-value \\
\hline$X$ & 1.585 & 1.567 & -0.1024 & $3.15 \cdot 10^{-5}$ & 105.799922 & 0.1756 \\
$Y$ & 1.504 & 1.567 & 0.1592 & $2.16 \cdot 10^{-5}$ & 21.043174 & 0.6437 \\
\hline
\end{tabular}

Applying (3.5) for the two-dimensional case, density functions of GPS signals are formulated as follows.

for $(x, y) \in \mathbb{R}^{2}$.

$$
\begin{gathered}
f_{(X, Y)}(x, y)=\exp \left(\frac{-\left[\Phi^{-1}\left(F_{X}(x)\right)\right]^{2}+2 \rho \Phi^{-1} F_{X}(x) \Phi^{-1} F_{Y}(y)-\left[\Phi^{-1} F_{Y}(y)\right]^{2}}{2\left(1-\rho^{2}\right)}\right) \\
\times \frac{f_{X}(x) f_{Y}(y)}{2 \pi\left[1-\rho^{2}\right]^{1 / 2} \varphi\left(\Phi^{-1} F_{X}(x)\right) \varphi\left(\Phi^{-1} F_{Y}(y)\right)}
\end{gathered}
$$

\subsection{Multivariate stable distribution of stock market data}

This subsection models daily return data from 6 stocks AK (Akorn), AP (Apple), FA (Facebook), MI (Microsoft), WM (Walmart), and AM (Amazon), using a sample from 01 May 2014 to 01 May 2019 to imply observations downloaded from Nasdaq Finance. Continuously compounded percentage returns are considered, i.e. daily returns are measured by log-differences of closing pricing multiplied by 100. Descriptive statistics together with Kolmogorov - Smirnov for normal distribution of the univariate series are shown in Table 3 and the result for the univariate stable model estimation are presented in Table 4.

Table 3 indicates that all the series have univariate distributions statistically different from Gaussian distribution. In the meantime, Table 4 shows the series having univariate stable distributions with a common stability index $\bar{\alpha}=1.4885$ less than 2 , driving us to guess the 6-coordinates vector of daily returns have multivariate stable distribution. The preliminary exploratory analysis in Table 3 also presents all series being asymmetric, therefore it can be concluded that the vector of daily returns is not an elliptically contoured stable vector.

To check if the 6-coordinates vector of daily returns have multivariate stable distribution with Gaussian copula, applying the same argument as that presented in the subsection 3.2 , we used the function named gofCopula in the copula package, testing the hypothesis of owning Gaussian copula for the vector of daily returns. However, the calculation gave a p-value of 0.0004995 , rejecting the above hypothesis of Gaussian copula.

That phenomenon may be caused by the fact that the multivariate distribution of the 5 -years data is a "mixture" of several distributions of data collected in shorter periods, at the same time, the relationships between daily returns of any stock market are changing year - by - year. It is possible that vectors of daily returns in any one - year collected data have Gaussian copula even when their five-years "mixture" does not. In this context, we investigate one - year period's data, dividing each of the given daily returns series into samples from the one - year periods of $1 / 5 / 2014-1 / 5 / 2015,1 / 5 / 2015-1 / 5 / 2016$, $1 / 5 / 2016-1 / 5 / 2017,1 / 5 / 2017-1 / 5 / 2018$, and $1 / 5 / 2018-1 / 5 / 2019$. 
Table 3. Normal distribution test for Nasdaq stock market daily return data

\begin{tabular}{llllll}
\hline Daily return & Mean & SD & skew & kurtosis & p-value \\
\hline Akorn & -0.18 & 4.65 & -6.97 & 120.11 & $2.200 \times 10^{-16}$ \\
Amazon & 0.15 & 1.89 & 0.48 & 8.19 & $7.293 \times 10^{-10}$ \\
Apple & 0.07 & 1.53 & -0.33 & 4.35 & $5.181 \times 10^{-8}$ \\
Facebook & 0.09 & 1.80 & -0.89 & 19.78 & $1.354 \times 10^{-8}$ \\
Microsoft & 0.09 & 1.45 & 0.15 & 7.06 & $3.715 \times 10^{-7}$ \\
Walmart & 0.02 & 1.23 & -0.12 & 17.59 & $1.186 \times 10^{-7}$ \\
\hline
\end{tabular}

Table 4. K-S test for univariate stability of Nasdaq daily returns

\begin{tabular}{lllllll}
\hline Daily return & $\alpha$ & $\bar{\alpha}$ & $\beta$ & $\gamma$ & $\delta$ & $\mathrm{p}$-value \\
\hline Akorn & 1.323 & 1.4885 & -0.139 & 1.3814575 & 0.0928667 & 0.2145 \\
Amazon & 1.507 & 1.4885 & -0.136 & 0.8938552 & 0.1625179 & 0.3195 \\
Apple & 1.433 & 1.4885 & -0.112 & 0.7530880 & 0.0997931 & 0.5816 \\
Facebook & 1.564 & 1.4885 & -0.153 & 0.9040878 & 0.1411224 & 0.2961 \\
Microsoft & 1.495 & 1.4885 & -0.024 & 0.7038934 & 0.0739206 & 0.5166 \\
Walmart & 1.609 & 1.4885 & -0.150 & 0.6042559 & 0.0620062 & 0.5488 \\
\hline
\end{tabular}

Table 5. K-S tests for normal distribution of Nasdaq daily returns

\begin{tabular}{lllllll}
\hline Period & $\begin{array}{l}\text { AK } \\
(\mathrm{p} \text {-value })\end{array}$ & $\begin{array}{l}\text { AM } \\
(\mathrm{p} \text {-value })\end{array}$ & $\begin{array}{l}\text { AP } \\
(\mathrm{p} \text {-value })\end{array}$ & $\begin{array}{l}\text { FA } \\
(\mathrm{p} \text {-value })\end{array}$ & $\begin{array}{l}\text { MI } \\
(\mathrm{p} \text {-value })\end{array}$ & $\begin{array}{l}\text { WM } \\
(\mathrm{p} \text {-value })\end{array}$ \\
\hline $\mathbf{2 0 1 4 - 2 0 1 5}$ & 0.013261 & 0.008177 & 0.391102 & 0.744801 & 0.008314 & 0.115903 \\
\hline $\mathbf{2 0 1 5 - 2 0 1 6}$ & 0.018070 & 0.047730 & 0.039080 & 0.061702 & 0.049890 & 0.038790 \\
\hline $\mathbf{2 0 1 6 - 2 0 1 7}$ & 0.003373 & 0.120602 & 0.041780 & 0.115101 & 0.008741 & 0.008337 \\
\hline $\mathbf{2 0 1 7 - 2 0 1 8}$ & $2.2 / 10^{16}$ & 0.021290 & 0.103902 & 0.006812 & 0.011230 & 0.000813 \\
\hline $\mathbf{2 0 1 8 - 2 0 1 9}$ & $5.32 / 10^{8}$ & 0.004618 & 0.009243 & 0.000610 & 0.029810 & 0.169003 \\
\hline
\end{tabular}

The Kolmogorov - Smirnov tests checking the normal distribution hypothesis for each daily returns series of AK; AM; AP; FA; MI; and WM for each one - year period, provide the correspond p-values given in Table 5. Almost all p-values smaller than $5 \%$ confirm the significant divergence from normal distribution of the 6-dimensional vector. Simultaneously, the greater than 5\% p-values of Kolmogorov - Smirnov tests in Table 6 are crucial arguments to conclude all the daily returns series of AK; AM; AP; FA; MI; and WM have univariate stable distributions with common stable index $\bar{\alpha}$.

After the above conclusion, we guess the 6-components vectors of one - year series of AK; AM; AP; FA; MI; and WM have multivariate stable distributions with stable index $\bar{\alpha}$ (the average number of $\alpha$ 's of those returns series in each one - year period). The values of parameter $\beta$ in Table 6 show almost all the series are asymmetric, then the 6 -coordinate vectors of one - year daily returns are not elliptically contoured stable vectors. Therefore the method proposed by Nolan[12] for modeling density functions of stable random vectors cannot be applied to these data.

To solve the problem, model of multivariate stable distributions with Gaussian copula was used. In the first step, the correlation matrices of daily returns for each year (after normalizing by respective functions determined in Corollary 2.2) were calculated, with results given in Table 7 (showing only upper diagonal part of each correlation matrix). 
Table 6. K-S test for univariate stability of Nasdaq daily returns

\begin{tabular}{|c|c|c|c|c|c|c|c|c|}
\hline Period & return & Size & $\alpha$ & $\bar{\alpha}$ & $\beta$ & $\gamma$ & $\delta$ & p-value \\
\hline \multirow{6}{*}{$\begin{array}{l}2014- \\
2015\end{array}$} & AK & 253 & 1.585 & 1.749 & -0.119 & 1.5530655 & 0.2911653 & 0.8921 \\
\hline & $\mathrm{AM}$ & 253 & 1.890 & 1.749 & .250 & & & 0.9705 \\
\hline & $\mathbf{A P}$ & 253 & 1.816 & 1.749 & 0.226 & 0.9143218 & 0.0940329 & 0.2943 \\
\hline & FA & 253 & 1.834 & 1.749 & -0.487 & 1.0605608 & 0.1819212 & 0.1691 \\
\hline & MI & 253 & 1.802 & 1.749 & 0.950 & & -0.0944571 & 0.8333 \\
\hline & WM & 253 & 1.566 & 1.749 & -0.164 & 0.5259360 & 0.0202864 & 0.6175 \\
\hline \multirow{6}{*}{$\begin{array}{l}2015- \\
2016\end{array}$} & AF & 253 & .520 & .594 & .129 & 8297 & 622 & 0.9705 \\
\hline & $\mathbf{A M}$ & 253 & & 1.594 & .170 & & & 2943 \\
\hline & $\mathbf{A P}$ & 253 & 464 & 1.594 & 011 & & -0. & 6175 \\
\hline & FA & 253 & & 1.594 & -0.275 & & & 175 \\
\hline & MI & 253 & & & -0.279 & & & 8921 \\
\hline & WM & 253 & 1.735 & 1.594 & -0.342 & 0.7393400 & -0.0387758 & 0.6175 \\
\hline \multirow{6}{*}{$\begin{array}{l}2016- \\
2017\end{array}$} & AK & 252 & 1.386 & 1.542 & -0.052 & 1.4137946 & 0.0060832 & 0.5412 \\
\hline & $\mathbf{A M}$ & 252 & 1.571 & 1.542 & 0.040 & & & 412 \\
\hline & $\mathbf{A P}$ & 252 & 1.554 & 1.542 & 0.052 & & & 900 \\
\hline & FA & 252 & & 1.542 & -0.059 & & & 5412 \\
\hline & MI & 252 & & 1.542 & 0162 & & & 0.6900 \\
\hline & WM & 252 & 1.587 & 1.542 & 0.013 & 0.4897892 & 0.0414158 & 0.9971 \\
\hline \multirow{6}{*}{$\begin{array}{l}2017- \\
2018\end{array}$} & & 253 & 879 & 416 & 0.022 & 1033 & 055 & 0.2467 \\
\hline & $\mathrm{AM}$ & 253 & & 1.416 & -0.242 & & & 0.7655 \\
\hline & $\mathbf{A P}$ & 253 & 1.519 & 1.416 & -0.272 & 8885 & & 0.8333 \\
\hline & FA & 253 & 1.449 & 1.416 & -0.226 & 0.7877504 & 0.1023312 & 0.6175 \\
\hline & MI & 253 & 1.383 & 1.416 & -0.206 & 0.6377951 & 0.1471863 & 0.1690 \\
\hline & WM & 253 & 1.672 & 1.416 & -0.349 & 0.6182917 & 0.1956893 & 0.6924 \\
\hline \multirow{6}{*}{$\begin{array}{l}2018- \\
2019\end{array}$} & & 52 & 5 & 1.486 & 92 & & & 0.9375 \\
\hline & $\mathbf{A M}$ & 252 & & & & 26232 & & \\
\hline & $\mathbf{A P}$ & 252 & & & -0.076 & 0.8866676 & 0.2004820 & 0.7634 \\
\hline & FA & 252 & & & 0.163 & & & 0.2447 \\
\hline & MI & 252 & 1.363 & 1.486 & -0.098 & 0.8032993 & 0.2114007 & 0.615 \\
\hline & WM & 252 & 1.696 & 1.486 & -0.420 & 0.6659449 & 0.0687611 & 0.4709 \\
\hline
\end{tabular}

Then, the function named gofCopula in the copula package was used to test the hypotheses of having Gaussian copula for each one - year 6-coordinates vector of daily returns. The calculations generated the p-values of $0.09241 ; 0.4001 ; 0.4261 ; 0.03247$; and 0.1424 for the periods of $1 / 5 / 2014-1 / 5 / 2015,1 / 5 / 2015-1 / 5 / 2016,1 / 5 / 2016-1 / 5 / 2017,1 / 5 / 2017$ $1 / 5 / 2018$, and $1 / 5 / 2018-1 / 5 / 2019$, respectively. The result shows that the copula of daily returns vector is significantly different from Gaussian copula only for the period of $1 / 5 / 2017-1 / 5 / 2018$. Consequently, for other periods, (3.5) can be applied to determine the density functions of 6 -coordinates vector of daily returns.

In particular, the density functions are defined by the following explicit form:

$$
\begin{gathered}
f_{\mathbf{Y}_{i}}\left(y_{1}, \ldots, y_{6}\right)= \\
=\frac{\exp \left(-\frac{1}{2}\left(\Phi^{-1} F_{S_{i 1}}\left(y_{1}\right), \ldots,\right.\right.}{\left.\left.\Phi^{-1} F_{S_{i 6}}\left(y_{6}\right)\right) \Sigma_{i}^{-1}\left(\Phi^{-1} F_{S_{i 1}}\left(y_{1}\right), \ldots, \Phi^{-1} F_{S_{i 6}}\left(y_{6}\right)\right)^{T}\right)} \\
(2 \pi)^{3}\left|\Sigma_{i}\right|^{1 / 2} \varphi\left(\Phi^{-1} F_{S_{i 1}}\left(y_{1}\right)\right) \ldots \varphi\left(\Phi^{-1} F_{S_{i 6}}\left(y_{6}\right)\right) \\
\times f_{S_{i 1}}\left(y_{1}\right) \ldots f_{S_{i 6}}\left(y_{6}\right) .
\end{gathered}
$$


Table 7. Correlation matrices of yearly Nasdaq daily returns

\begin{tabular}{llllllll}
\hline Period & Return & AK & AM & AP & FA & MI & WM \\
\hline & AK & 1 & 0.2186643 & 0.2475693 & 0.3077665 & 0.2459316 & 0.1601064 \\
$\mathbf{2 0 1 4}-$ & AM & - & 1 & 0.3409933 & 0.4551869 & 0.3825091 & 0.3096944 \\
$\mathbf{2 0 1 5}$ & AP & - & - & 1 & 0.4335172 & 0.3217411 & 0.3265398 \\
$\left(\Sigma_{1}\right)$ & FA & - & - & - & 1 & 0.3563987 & 0.1559727 \\
& MI & - & - & - & - & 1 & 0.4056158 \\
& WM & - & - & - & - & - & 1 \\
\hline & AK & 1 & 0.3588723 & 0.2654328 & 0.2849989 & 0.3524828 & 0.1686205 \\
$\mathbf{2 0 1 5}-$ & AM & - & 1 & 0.4593876 & 0.6691572 & 0.5978800 & 0.2696340 \\
$\mathbf{2 0 1 6}$ & AP & - & - & 1 & 0.4907881 & 0.6187722 & 0.3225324 \\
$\left(\Sigma_{2}\right)$ & FA & - & - & - & 1 & 0.6049646 & 0.2915228 \\
& MI & - & - & - & - & 1 & 0.3800727 \\
& WM & - & - & - & - & - & 1 \\
\hline & AK & 1 & 0.1343061 & 0.1724847 & 0.1420178 & 0.1304932 & 0.0985428 \\
$\mathbf{2 0 1 6}-$ & AM & - & 1 & 0.3852057 & 0.6213989 & 0.5295887 & 0.2304948 \\
$\mathbf{2 0 1 7}$ & AP & - & - & 1 & 0.4696930 & 0.3770917 & 0.1327082 \\
$\left(\Sigma_{3}\right)$ & FA & - & - & - & 1 & 0.4943592 & 0.1408454 \\
& MI & - & - & - & - & 1 & 0.1764853 \\
& WM & - & - & - & - & - & 1 \\
\hline & AK & 1 & 0.1331431 & 0.1339723 & 0.0957475 & 0.2203172 & 0.1150943 \\
$\mathbf{2 0 1 7}$ & AM & - & 1 & 0.5391196 & 0.5924327 & 0.6516110 & 0.1614915 \\
$\mathbf{2 0 1 8}$ & AP & - & - & 1 & 0.4973002 & 0.6237823 & 0.2566277 \\
$\left(\Sigma_{4}\right)$ & FA & - & - & - & 1 & 0.5590985 & 0.1808916 \\
& MI & - & - & - & - & 1 & 0.2446375 \\
& WM & - & - & - & - & - & 1 \\
\hline & AK & 1 & 0.1580187 & 0.0902602 & 0.0766932 & 0.0922525 & 0.1139641 \\
$\mathbf{2 0 1 8}$ & AM & - & 1 & 0.6874822 & 0.5985045 & 0.7364774 & 0.2353871 \\
$\mathbf{2 0 1 9}$ & AP & - & - & 1 & 0.4927705 & 0.6563119 & 0.1773758 \\
$\left(\Sigma_{5}\right)$ & FA & - & - & - & 1 & 0.5133173 & 0.0338976 \\
& MI & - & - & - & - & 1 & 0.2756855 \\
& WM & - & - & - & - & - & 1 \\
\hline & & & & & & & \\
\hline
\end{tabular}

Where $i=1,2,3$, and 5 for the one-year periods of $1 / 5 / 2014-1 / 5 / 2015,1 / 5 / 2015-1 / 5 / 2016$, $1 / 5 / 2016-1 / 5 / 2017$, and 1/5/2018-1/5/2019, respectively. Simultaneously, $F_{S_{i 1}}, \ldots, F_{S_{i 6}}$ and $f_{S_{i 1}}, \ldots, f_{S_{i 6}}$ are univariate cumulative distribution functions and density functions of daily returns series of AK; AM; AP; FA; MI; and WM with stable parameters $(\bar{\alpha}, \beta, \gamma, \delta)$ given in Table 6 , whilst $\Sigma_{i}$ is the correlation matrix presented in Table 7.

In short, the multivariate stable density functions can be directly used to compute the implied distribution of any portfolio of 6 assets AK; AM; AP; FA; MI; and WM. As the joint distribution of the vector of asset-returns is a multivariate stable distribution, the univariate distribution of returns of any portfolio of these assets is also stable. This approach can be used to solve many problems related to portfolio selection.

Acknowledgment. The research is funded by the Vietnam National Foundation for Sciences and Technology Development (NAFOSTED) under grant number 101.03-2017.07, and is partially supported by the International Center for Research and Postgraduate 
Training in Mathematics (ICRTM) under grant number ICRTM01-2020.03. Thanks are due to Dr. Bui Quang Nam for the sharing the GPS data used in the application part of this study.

\section{References}

[1] R. J. Adler, R. E. Feldman, M. S Taqqu. A Practical Guide to Heavy Tailed Data, Birkhäuser, Boston, 1998.

[2] N. Bui Quang. On stable probability distributions and statistical application, Thesis, Academy of military science and technology, Ha Noi, 2016.

[3] P. Embrechts, F. Lindskog, A. McNeil. Modelling Dependence with Copulas and Applications to Risk Management, Handbook of Heavy Tailed Distributions in Finance, 2003, ed. Rachev S., Elsevier, Chapter 8, 329-384, 2001.

[4] E. Fama. The behavior of stock prices, Journal of Business 38, 34-105, 1965.

[5] C. Genest, B. Rémillard. Validity of the parametric bootstrap for goodness-of-fit testing in semiparametric models, Annales de Institut Henri Poincaré - Probabilités et Statistiques 44 no. 6, 1096-1127, 2008.

[6] S. M. Kogon, D. B. Williams. Characteristic function based estimation of stable parameters, In Adler R., Feldman R. and Taqqu M. (eds.) A Practical Guide to Heavy Tailed Data, Birkhäuser, Boston, MA, 311-335, 1998.

[7] I. Kojadinovic, J. Yan. Modeling Multivariate Distributions with Continuous Margins Using the copula $R$ Package, J. Stat. Softw. 34 no. 9, 1-20, 2010.

[8] R. M. Kunst. Apparently stable increments in finance data: Could ARCH effects be the cause?, J. Statist. Comput. Simulation 45, 121-127, 1993.

[9] F. Lamantia, S. Ortobelli, S. Rachev. VaR, CVaR and Time Rules with Elliptical and Asymmetric Stable Distributed Returns, Investment Management and Financial Innovations 3, Issue 4, 19-39, 2006.

[10] J. H. McCulloch. Simple consistent estimators of stable distribution parameters, Comm. Statist. Simulation Comput. 15, 1109-1136, 1986.

[11] J. H. McCulloch. Financial applications of stable distributions, Handbook of Statistics 14, ed. G. Maddala and C. Rao, Elsevier Science Publishers, North-Holland, 393-425, 1996.

[12] J.P. Nolan. Multivariate elliptically contoured stable distributions: theory and estimation, Comput. Statist. 28, Issue 5, 2067-2089, 2013.

[13] J.P. Nolan. Stable Distributions - Models for Heavy Tailed Data, Birkhauser: Boston, MA, USA, 2016.

[14] K. J. Palmer, M. S. Ridout, B. J. T. Morgan. Modelling cell generation times using the tempered stable distribution, J. R. Stat. Soc. Ser. C. Appl. Stat. 57, 379-397, 2008.

[15] G. Samorodnitsky, M. S. Taqqu. Stable Non-Gaussian Random Processes, New York, NY, Chapman \& Hall, 1994.

[16] P. Samuelson. Efficient portfolio selection for Pareto - Lévy investments, J. Financ. Quant. Anal. 2, 107-117, 1967.

[17] A. Sklar. Fonctions de rèpartition à $n$ dimensions et leurs marges. Publications de l'Institut de Statistique de l'Universitè de Paris 8, 229-231, 1959.

[18] M. S. Taqqu. The modeling of Ethernet data and of signals that are heavy-tailed with infinite variance. Scand. J. Stat. 829, 273-295, 2002. 